\title{
A minimally invasive pericoccygeal approach to resection of a large presacral schwannoma: case report
}

\author{
Osa Emohare, MBBS, PhD, Molly Stapleton, MS, PA-C, and Alejandro Mendez, MD \\ Department of Neurosurgery, Regions Hospital, St. Paul, Minnesota
}

\begin{abstract}
Resection of large presacral schwannomas can present a challenge. The posterior approach is commonly associated with coccygeal disarticulation, partial sacral resection, and muscular disarticulation, which can all result in significant morbidity. Minimally invasive surgery may obviate some of the morbidity traditionally associated with this approach. The authors present the case of a morbidly obese 49 -year-old man with an enlarging presacral schwannoma. The patient refused laparoscopic resection because of the morbidity he had experienced with a previous laparoscopic surgery. The tumor was resected using a minimally invasive paracoccygeal approach, which affords improved access with minimal morbidity.

http://thejns.org/doi/abs/10.3171/2014.11.SPINE14396
\end{abstract}

KEY WORDS disarticulation; laparoscopic surgery; presacral schwannoma; surgical procedures; minimally invasive; sacral

$\mathrm{S}$ CHWANNOMAS in the presacral space are a rare variant of these neural tumors; ${ }^{6,9}$ by virtue of their location, they can cause a variety of symptoms related mainly to their mass effect. They are most often resected laparoscopically or, in the case of larger masses, by laparotomy. ${ }^{13}$ Unique patient circumstances, such as morbid obesity, risk of hemorrhage, and previous morbidity, can preclude these "traditional" approaches to resection. We report the case of a presacral schwannoma resected via a minimally invasive paracoccygeal exposure using tubular retractors and endoscopy - an approach not previously described.

\section{Case Report}

History and Examination

A 49-year-old man with a history of worsening chronic low-back pain and difficulty urinating had a presacral, solid, homogeneously enhancing mass on MRI. A followup scan 3-months later revealed an increase in tumor size.
Located to the left of the midline at the junction of S-1 and $\mathrm{S}-2$, the tumor had enlarged to $8 \mathrm{~cm}$ in diameter (Fig. 1).

The patient's medical history included hypertension, Type 2 diabetes mellitus, morbid obesity (body mass index $41.5 \mathrm{~kg} / \mathrm{m}^{2}$ ), previous bilateral L4-5 and L5-S1 rhizolysis, and a previous laparoscopic cholecystectomy. Given his body habitus, a laparoscopic transperitoneal approach was recommended, but morbidity from a previous laparoscopic surgery led the patient to refuse this approach. Considering the anatomical location of the tumor, the shortest route to the lesion was a pericoccygeal approach, which would be easiest to perform using a minimally invasive method with tubular retractors. Moreover, the patient's religion precluded the use of blood products since he was a Jehovah's Witness.

\section{Operation}

Informed consent was obtained, which included the possibility of converting to an open procedure, if needed. The patient was anesthetized, intubated, and placed in the 

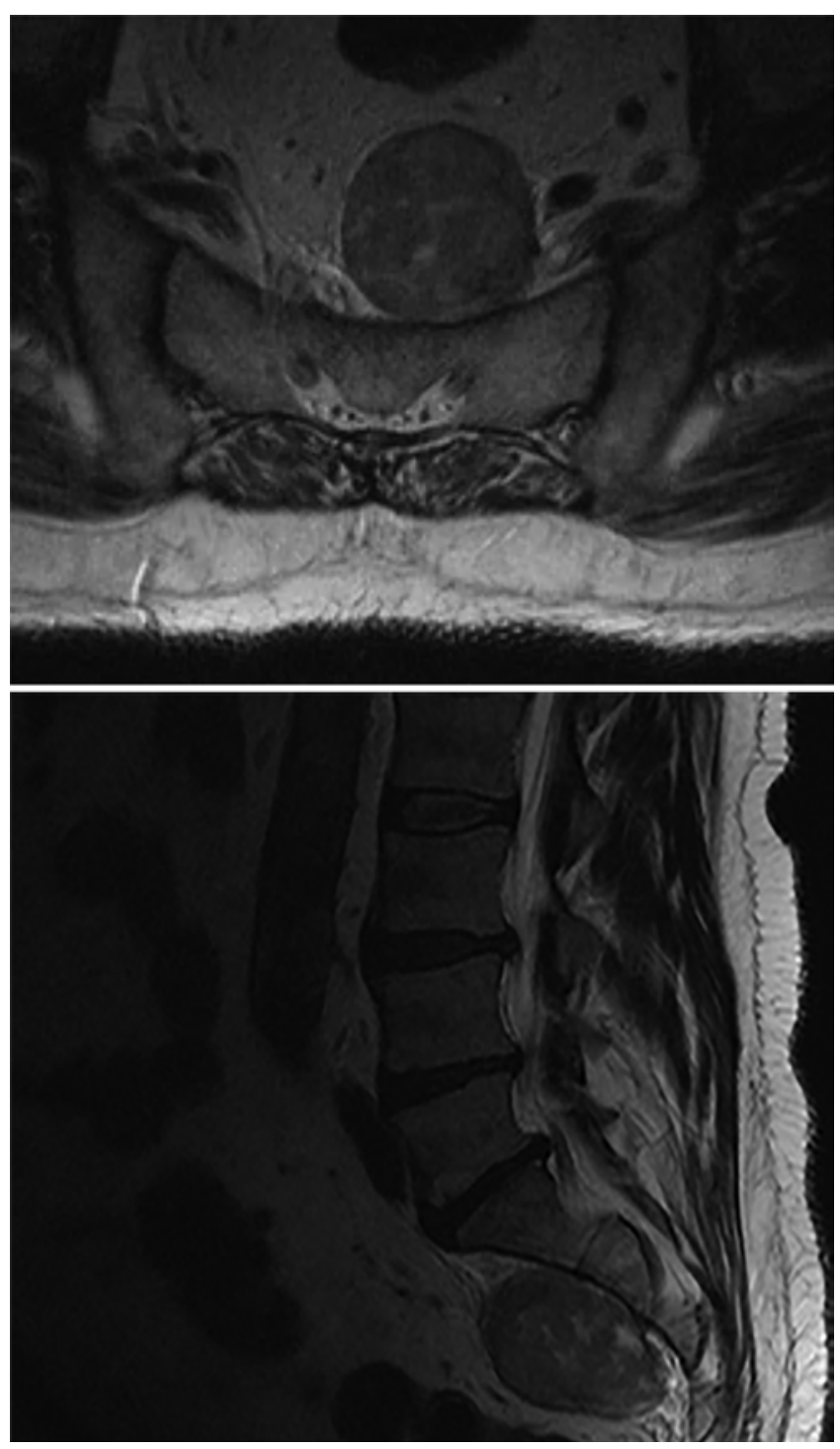

FIG. 1. Axial (upper) and sagittal (lower) preoperative MR images of a mass lesion located in the presacral space.

lateral decubitus position, with his right side up. Anteroposterior and lateral fluoroscopy was used to guide the location of the incision, which was about $6 \mathrm{~cm}$ long in the pericoccygeal approach and about $4 \mathrm{~cm}$ lateral to the anus and 3-4 cm cephalic to it. The sacro-pelvic and coccygealpelvic ligaments were exposed. The inferior fibers of these ligaments were released, and with gentle blunt dissection, a surgical corridor was created in the presacral space, anterior and cephalic to the ligaments. Dilators were advanced, a Medtronic direct lateral interbody fusion retractor system (Medtronic Sofamor Danek) was positioned, and the retractor was secured to a table-mounted holder. Neuromonitoring and a direct nerve stimulator were used during this case. Prior to any cut or dissection, we would stimulate so as not to damage any nerve roots.

The tubular retractor was slightly opened, allowing further visualization of the inferior right-sided margin of the tumor. We commenced endoscopic resection using a $0^{\circ}$ high-definition rhinoscope. The tumor had the characteristic rubbery consistency of a schwannoma. It was cored using endoscopic instruments, and electrocautery scissors were used to cut through the capsule. Pituitary rongeurs were used to core the center of the tumor, and dissectors were used to develop the tumor margin. During surgery, we were able to stimulate the S-1 nerve root where the tumor originated. The margins were clear, with a good plane and no evidence of infiltration; they were peeled away, and brisk bleeding was encountered from a branch of the iliac vein. Hemostasis was ultimately obtained with bipolar cautery, compression, and Floseal hemostatic matrix (Baxter Inc.). The patient remained hemodynamically stable throughout. Once the bleeding was completely controlled, residual edges of the tumor were removed and the excision was completed. A drain was placed at the excision site, and the wound was closed in layers. Total surgical time was 4 hours 9 minutes. Estimated blood loss was $1300 \mathrm{ml}$. The patient awoke with no neurological deficits.

\section{Postoperative Course}

His postoperative course was uneventful; he maintained a hemoglobin level $>11 \mathrm{~g} / \mathrm{dl}$ and was ambulating on postoperative Day 1. The drain was removed, and the patient was discharged on postoperative Day 4. Subsequent pathology revealed that the tumor stained diffusely positive for S100 and was negative for smooth muscle actin and CD34, and a low Ki 67 index was noted, in keeping with a diagnosis of schwannoma. Postoperative MRI showed gross-total resection with some peri-resection site changes. The patient had a rapid recovery with no complications. Follow-up MRI at 12 months showed the resolution of all postoperative changes, with no evidence of residual tumor (Fig. 2).

\section{Discussion}

Although schwannomas are commonly slow growing and painless, the mass effect of presacral schwannomas usually constitutes the surgical indication. Patients with these lesions are most often in their 2nd to 5th decades of life and tend to be female. ${ }^{13,15}$ In the absence of a syndromic component to the tumor, such as von Recklinghausen's disease, recurrence or malignant transformation is unlikely, meaning that extensive resection is curative., ${ }^{2,13}$ The lack of invasive growth exhibited by these tumors and their thick capsular lining make presacral schwannomas amenable to complete resection. ${ }^{4,79}$ Preoperative biopsy was not performed in our featured case given the benign-appearing features of the tumor, although there are divergent views regarding biopsy for more atypical tumors. ${ }^{1}$ Generally, 3 potential approaches have been described for presacral tumor resection: anterior or abdominal (whether open or laparoscopic), posterior, or a combination. ${ }^{3,14,17}$ Resection of these tumors is increasingly performed using laparoscopic techniques, ${ }^{12,17}$ although open laparotomy may be performed in the case of larger tumors. ${ }^{14} \mathrm{~A}$ more recent series advocated a posterior approach to avoid the abdominal morbidity associated with 

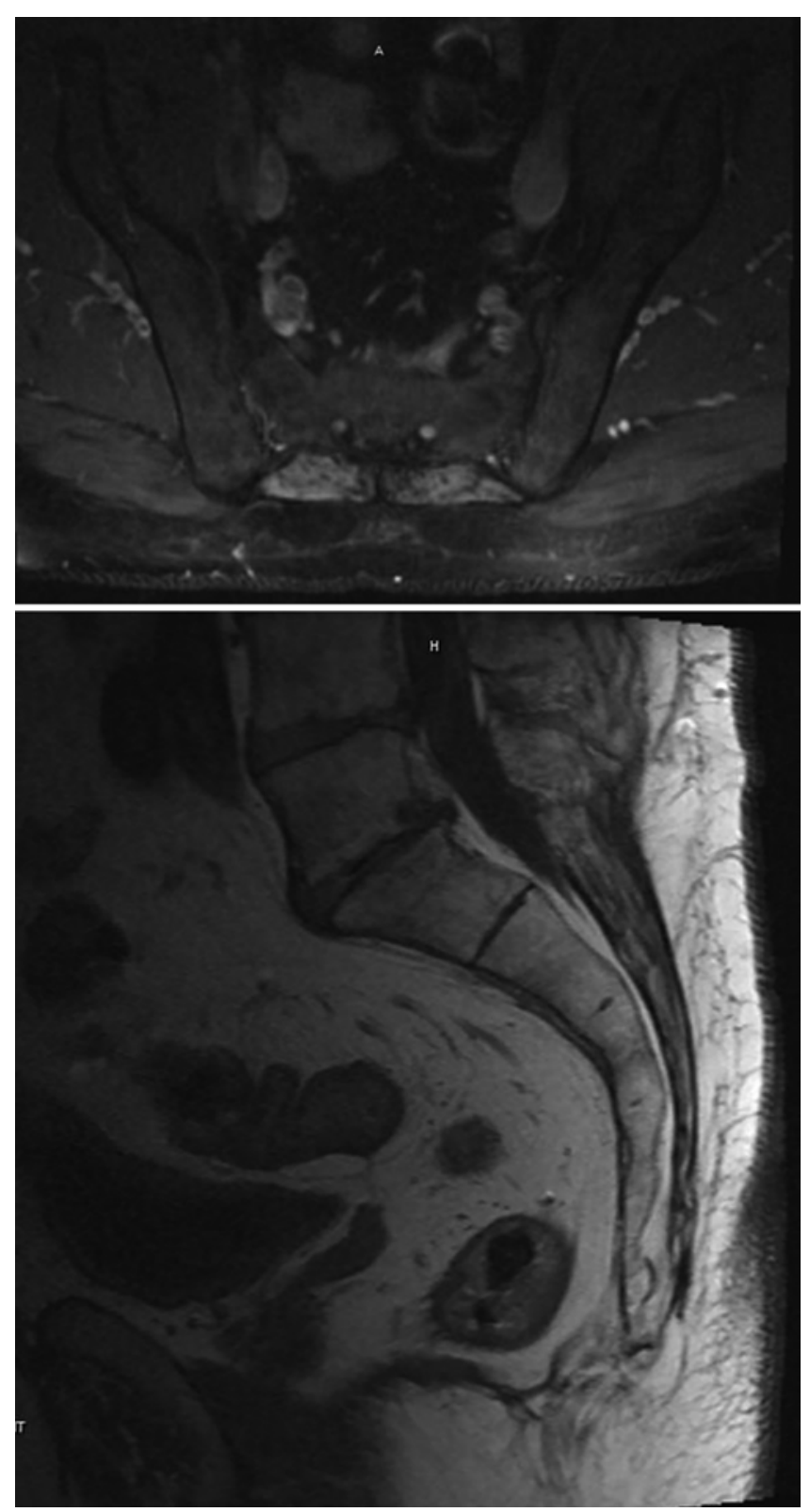

FIG. 2. Axial (upper) and sagittal (lower) MR images obtained at 12 months postoperatively, showing the absence of a mass lesion.

an anterior approach. ${ }^{14}$ Although the tumors presented in that series were broadly comparable with the current case in terms of their dimensions, most cases seemed to require disarticulation of the coccyx and resection of part of the sacrum. The posterior approach, in which a patient is placed in the prone jackknife position, with excision of the coccyx, can be associated with significant perioperative morbidity; in this context, extensive sacral resection is not uncommon and has an attendant potential for functional impairment. ${ }^{5,8,10,11}$ With this approach, neurological dysfunction, especially neurogenic bladder, is the most frequently reported complication, ${ }^{5,10,11}$ although infective complications, hemorrhage, dysesthesia, and stool incontinence have also been encountered. ${ }^{11}$ It has been report- ed that lower sacral resections are associated with fewer complications, as no permanent neurological morbidity has occurred in patients whose sacral resection was below S-2. ${ }^{5}$ The coccyx may be disarticulated either alone or with the attachment of the gluteus maximus; when this happens, it is usually in combination with a sacrectomy at the level of S4-5.,16

The utility of the paracoccygeal approach has been examined, ${ }^{3}$ as has the potential for detaching the coccyx, gluteus maximus, and part of the sacrum. The patients and cadavers studied were positioned in either the prone jackknife position or a lithotomy position. In addition, the anococcygeal ligament and the levator ani muscle were divided to enter the supra-levator space. ${ }^{3}$ The minimally invasive paracoccygeal approach we present may reduce the need for bony resection and is especially germane because the patient's religious circumstances limited the options for managing morbidity or hemodynamic instability.

In this report, we presented a less destructive, less invasive approach to these tumors. Because of the patient's morbid obesity, the transabdominal route was not ideal, and he opposed laparoscopy. With the tumor located high in the presacral space, the traditional posterior approach would have required extensive sacral resection. Our choice of directional approach was made because it is easier to address pathology on the opposite ventral aspect of the sacrum, crossing the midline. This provides a view perpendicular to the apex of the tumor as opposed to the tangential view obtained using an ipsilateral approach (Figs. 3 and 4). The paracoccygeal approach has been refined over the last 10-15 years with the introduction of the axial presacral lumbar interbody fusion system (AxiaLIF, TranS1 Inc.). This approach provides quick, minimally invasive access to the anterior junction of S1-2 with less morbidity while the patient is prone. Modifications we made to this technique included putting the patient in a lateral decubitus position to allow the viscera to fall forward, away from the resection site, and to decrease venous congestion. We worked from the right side across the anterior surface of the sacrum, with the tumor on the left side, which provided a panoramic view of the inferior and medial aspects of the tumor and allowed full visualization by simply modifying the position of the retractor system a few degrees at a time.

As in most minimally invasive endoscopic resections, the tumor excision technique is different from the standard open approach. Here, the idea is to core the center of the tumor as much as possible and then to let the thin residual skin collapse inward, only then creating a plane between the tumor and adjacent tissues. In our patient, the resection went extraordinarily well until we created a vascular injury with significant blood loss. This is something anyone attempting this technique should be aware of. It took some time to control, working through a port $23 \mathrm{~mm}$ in diameter and $15 \mathrm{~cm}$ long. Ultimately, we were able to control the bleeding with no harm to the patient. When bleeding is encountered, possible approaches to achieve hemostasis include using laparoscopic hemostatic clips, long bipolar probes, electrocautery laparoscopic scissors, and laparoscopic or thoracoscopic harmonic scalpels. The first 3 tools were used in the present case. Postoperative 


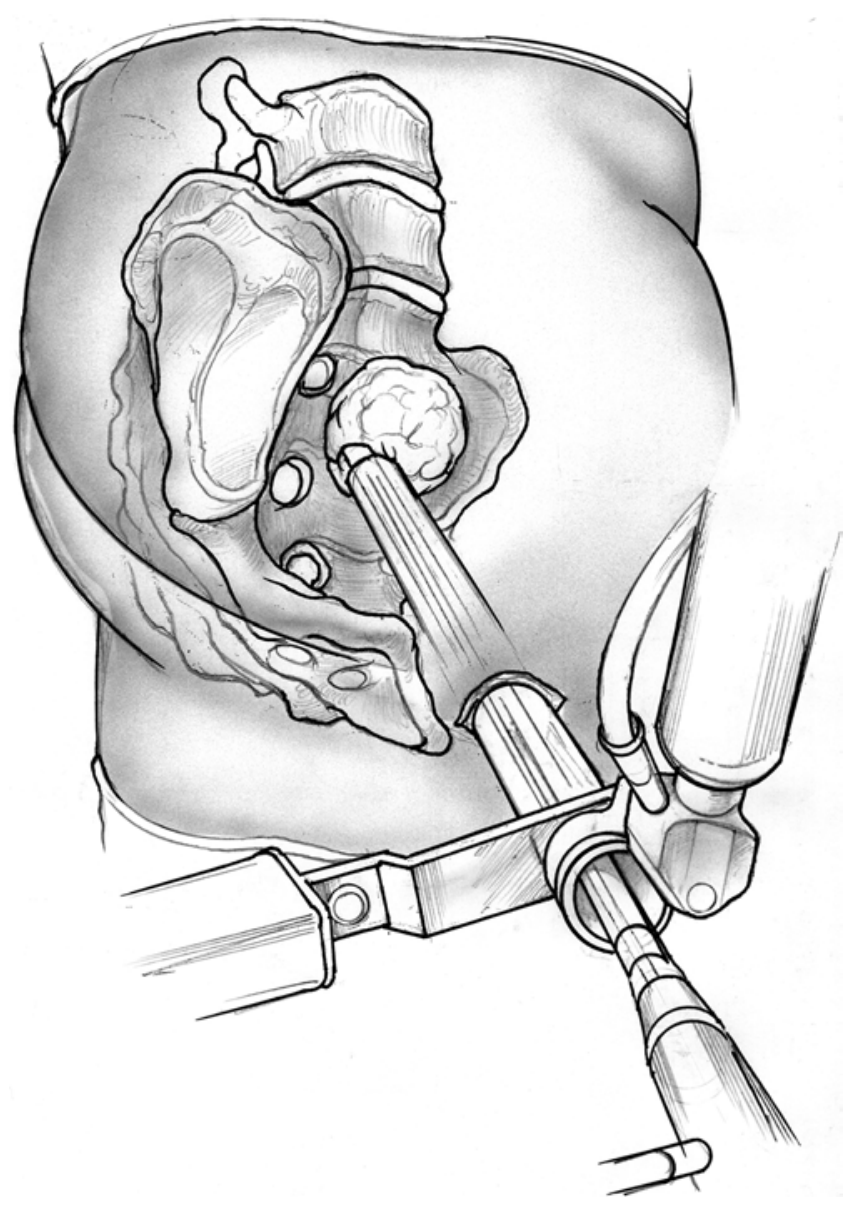

FIG. 3. Illustration of surgical approach to the tumor. Copyright Medical Surgical Illustration. Published with permission.

imaging at 3 and 12 months demonstrated patency in the iliac vein.

This technique remains in its infancy and requires further development, such as the use of a laparoscopic harmonic scalpel or laparoscopic vascular clips. A current limitation is patient selection, as it is not appropriate for patients with previous surgery in the area, those with perirectal pathology including Crohn's disease, potentially those with ulcerative colitis, and those with abnormal vascular anatomy. Finally, the technique provides a limited view of the sacral foramina, thus limiting its application for extraforaminal tumors. Despite these shortcomings, we believe it is an exciting and potentially very useful approach to presacral pathology.

\section{Conclusions}

In summary, presacral schwannoma is an uncommon pathology that, in most cases, is resected abdominally. With appropriate patient selection, a minimally invasive paracoccygeal approach may offer minimal morbidity and relatively good access - especially in morbidly obese patients and those with multiple comorbid conditions.

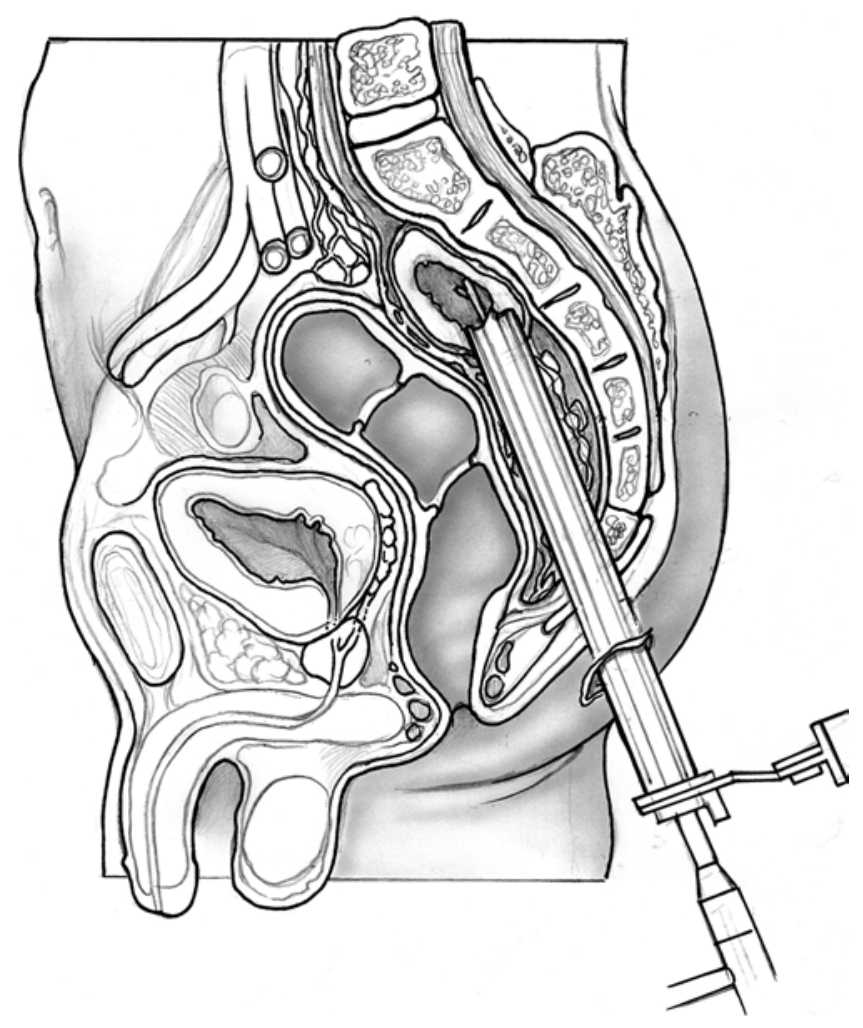

FIG. 4. Illustration of the anatomical relations of the tumor to neighboring structures. Copyright Medical Surgical Illustration. Published with permission.

\section{References}

1. Bennert KW, Abdul-Karim FW: Fine needle aspiration cytology vs. needle core biopsy of soft tissue tumors. A comparison. Acta Cytol 38:381-384, 1994

2. Brady KA, McCarron JP Jr, Vaughan ED Jr, Javidian P: Benign schwannoma of the retroperitoneal space: case report. J Urol 150:179-181, 1993

3. Buchs N, Taylor S, Roche B: The posterior approach for low retrorectal tumors in adults. Int J Colorectal Dis 22:381385,2007

4. Canelles E, Roig JV, Cantos M, García Armengol J, Barreiro E, Villalba FL, et al: [Presacral tumors. Analysis of 20 surgically treated patients.] Cir Esp 85:371-377, 2009 (Span)

5. Cody HS III, Marcove RC, Quan SH: Malignant retrorectal tumors: 28 years' experience at Memorial Sloan-Kettering Cancer Center. Dis Colon Rectum 24:501-506, 1981

6. Daneshmand S, Youssefzadeh D, Chamie K, Boswell W, Wu N, Stein JP, et al: Benign retroperitoneal schwannoma: a case series and review of the literature. Urology 62:993-997, 2003

7. Dozois EJ, Jacofsky DJ, Dozois RR: Presacral tumors, in Wolff BG, Fleshman JW, Beck DE, et al (eds): The ASCRS Textbook of Colorectal Surgery. New York: Springer, 2007, pp 501-514

8. Hassan I, Wietfeldt ED: Presacral tumors: diagnosis and management. Clin Colon Rectal Surg 22:84-93, 2009

9. Hughes MJ, Thomas JM, Fisher C, Moskovic EC: Imaging features of retroperitoneal and pelvic schwannomas. Clin Radiol 60:886-893, 2005

10. Jackson RJ, Gokaslan ZL: Spinal-pelvic fixation in patients with lumbosacral neoplasms. J Neurosurg 92 (1 Suppl):61-70, 2000 
11. Jao SW, Beart RW Jr, Spencer RJ, Reiman HM, Ilstrup DM: Retrorectal tumors. Mayo Clinic experience, 1960-1979. Dis Colon Rectum 28:644-652, 1985

12. Konstantinidis K, Theodoropoulos GE, Sambalis G, Georgiou M, Vorias M, Anastassakou K, et al: Laparoscopic resection of presacral schwannomas. Surg Laparosc Endosc Percutan Tech 15:302-304, 2005

13. Mastoraki A, Toska F, Tsiverdis I, Kyriazi M, Tsagkas A, Danias N, et al: Retroperitoneal schwannomas: dilemmas in diagnostic approach and therapeutic management. J Gastrointest Cancer 44:371-374, 2013

14. Samarakoon L, Weerasekera A, Sanjeewa R, Kollure S: Giant presacral schwannoma presenting with constipation: a case report. J Med Case Reports 6:285, 2012

15. White W, Shiu MH, Rosenblum MK, Erlandson RA, Woodruff JM: Cellular schwannoma. A clinicopathologic study of 57 patients and 58 tumors. Cancer 66:1266-1275, 1990

16. Wolpert A, Beer-Gabel M, Lifschitz O, Zbar AP: The management of presacral masses in the adult. Tech Coloproctol 6:43-49, 2002
17. Yang CC, Chen HC, Chen CM: Endoscopic resection of a presacral schwannoma. Case report. J Neurosurg Spine 7:86-89, 2007

\section{Author Contributions}

Conception and design: Emohare, Mendez. Acquisition of data: all authors. Analysis and interpretation of data: Emohare, Mendez. Drafting the article: Emohare. Critically revising the article: Emohare, Mendez. Reviewed submitted version of manuscript: Emohare, Mendez. Approved the final version of the manuscript on behalf of all authors: Emohare. Administrative/techni$\mathrm{cal} / \mathrm{material}$ support: all authors. Study supervision: Mendez.

\section{Correspondence}

Osa Emohare, Department of Neurosurgery, Regions Hospital, 640 Jackson St., St. Paul, MN 55101. email: oemohare@umn. edu. 